Pacific Journal of 


\title{
ON THE TENSOR PRODUCTS OF VON NEUMANN ALGEBRAS
}

\author{
JUN TOMIYAMa
}

Let $A$ and $B$ be $C^{*}$-algebras and let $A \otimes_{\alpha} B$ be their $C^{*}$-tensor product with Turumaru's $\alpha$-norm. The author has previously defined mappings $R_{\varphi}: A \otimes_{\alpha} B \rightarrow B$ and $L_{\psi}: A \otimes_{\alpha} B \rightarrow A$ via bounded linear functionals $\varphi$ on $A$ and $\psi$ on $B$, as follows :

$$
\begin{aligned}
& R_{\varphi}\left(\sum_{i=1}^{n} a_{i} \otimes b_{i}\right)=\sum_{i=1}^{n}\left\langle a_{i}, \varphi>b_{i},\right. \\
& L_{\varphi}\left(\sum_{i=1}^{n} a_{\imath} \otimes b_{i}\right)=\sum_{i=1}^{n}\left\langle b_{i}, \psi>a_{i},\right.
\end{aligned}
$$

and has shown how the families $\left\{R_{\varphi} \mid \varphi \in A^{*}\right\}$ and $\left\{L_{\psi} \mid \psi \in B^{*}\right\}$ determine the structure of the tensor product of $A$ and $B$. Moreover, in a joint paper with J. Hakeda the author also proved the existence of these kinds of mappings in tensor products of von Neumann algebras and gave some of their applications. Further applications of these mappings are shown in the present paper.

Theorem 2 says that the product $M \otimes N$ has property $L$ if one of the factors $M$ or $N$ has property $L$. This answers a question of Sakai. It can be shown that the above families of mappings determine completely the tensor products of von Neumann algebras (Theorem 3). Theorem 4 shows that if $\pi_{1}$ and $\pi_{2}$ are projection of norm one from $M_{1}$ and $N_{1}$ to their subalgebras $M_{2}$ and $N_{2}$, then there exists, without assuming their $\sigma$-weak continuity, a projection of norm one $\pi$ from $M_{1} \otimes \mathscr{B}(K) \cap \mathscr{B}(H) \otimes N_{1}$ to $M_{2} \otimes \mathscr{B}(K) \cap \mathscr{B}(H) \otimes N_{2}$ such that $\pi(a \otimes b)=\pi_{1}(a) \otimes \pi_{2}(b)$, where $a \in M_{1}$ and $b \in N_{1}$.

We always denote by $M \otimes N$ the tensor product of the von Neumann algebras $M$ and $N$ and by $M \otimes_{\alpha} N$ their tensor product as $C^{*}$-algebras. $M^{*}$ means the conjugate space of $M$ and $M_{*}$ the predual of the von Neumann algebra $M$.

The following theorem is the basic result cited in the above introduction; it is a more precise version of Lemma 2.5 of [1]. We give the proof for the sake of completeness.

Theorem 1. Let $M$ and $N$ be von Neumann algebras and $M \otimes N$ their tensor product. Then for each $\varphi \in M_{*}$ (resp. $\psi \in N_{*}$ ) there exists a $\sigma$-weakly continuous mapping $R_{\bullet}: M \otimes N \rightarrow N$ (resp. $\left.L_{\psi}: M \otimes N \rightarrow M\right)$ satisfying the following conditions: 


$$
\begin{gathered}
R_{\varphi}\left(\sum_{i=1}^{n} a_{i} \otimes b_{i}\right)=\sum_{i=1}^{n}<a_{i}, \varphi>b_{i}, \\
\left(\operatorname{resp.} L_{\psi}\left(\sum_{i=1}^{n} a_{i} \otimes b_{i}\right)=\sum_{i=1}^{n}<b_{i}, \psi>a_{i}\right) . \\
\text { (2) } \quad R_{\varphi}((1 \otimes a) x(1 \otimes b))=a R_{\varphi}(x) b \text { for } x \in M \otimes N, \\
\text { (resp. } L_{\psi}((a \otimes 1) x(b \otimes 1))=a L_{\psi}(x) b . \\
\text { (3) } \quad<x, \varphi \otimes \psi>=\left\langle R_{\varphi}(x), \psi\right\rangle=\left\langle L_{\psi}(x), \varphi>\text { for } x \in M \otimes N .\right.
\end{gathered}
$$

Moreover, the families of mappings $\left\{R_{\varphi} \mid \varphi \in M_{*}\right\}$ and $\left\{L_{\psi} \mid \psi \in N_{*}\right\}$ are total in $M \otimes N$.

Proof. Let $\varphi \otimes \psi$ be the product functional of $\varphi$ and $\psi$ which is $\sigma$-weakly continuous in $M \otimes N$. Put $f_{\varphi, x}(\psi)=\langle x, \varphi \otimes \psi\rangle$ for $x \in M \otimes N$. Then $f_{\varphi, x}$ is clearly a bounded linear functional on $N_{*}$ and as $\left(N_{*}\right)^{*}=N$ there exists a element $R_{\varphi}(x)$ in $N$ such that

$$
\langle x, \varphi \otimes \psi\rangle=f_{\varphi, x}(\psi)=\left\langle R_{\varphi}(x), \psi\right\rangle \text {. }
$$

It is an easy verification by this definition that the mapping $R_{\varphi}: x \rightarrow R_{\varphi}(x)$ is a $\sigma$-weakly continuous linear mapping. Similarly, we get the mapping $L_{\psi}$ and it is easily seen that assertion 3 holds.

Next, take an element $\sum_{i=1}^{n} a_{i} \otimes b_{i}$; then

$$
\begin{aligned}
& \left\langle R_{\varphi}\left(\sum_{i=1}^{n} a_{i} \otimes b_{i}\right), \psi\right\rangle=\left\langle\sum_{i=1}^{n} a_{i} \otimes b_{i}, \varphi \otimes \psi\right\rangle \\
= & \sum_{i=1}^{n}\left\langle a_{i}, \varphi\right\rangle\left\langle b_{i}, \psi\right\rangle=\left\langle\sum_{i=1}^{n}\left\langle a_{i}, \varphi\right\rangle b_{i}, \psi\right\rangle \\
= & \left\langle\sum_{i=1}^{n}\left\langle b_{i}, \psi\right\rangle a_{i}, \varphi\right\rangle=\left\langle L_{\psi}\left(\sum_{i=1}^{n} a_{i} \otimes b_{i}\right), \varphi\right\rangle,
\end{aligned}
$$

which implies 1. From these relations, we get

$$
\begin{aligned}
& R_{\varphi}\left((1 \otimes a) \sum_{i=1}^{n} x_{i} \otimes y_{i}(1 \otimes b)\right)=R_{\varphi}\left(\sum_{i=1}^{n} x_{i} \otimes a y_{i} b\right) \\
= & \sum_{i=1}^{n}\left\langle x_{i}, \varphi>a y_{i} b=a\left(\sum_{i=1}^{n}<x_{i}, \varphi>y_{i}\right) b=a R_{\varphi}\left(\sum_{i=1}^{n} x_{i} \otimes y_{i}\right) b,\right.
\end{aligned}
$$

and since $R_{\varphi}$ is $\sigma$-weakly continuous, $R_{\varphi}((1 \otimes a) x(1 \otimes b))=a R_{\varphi}(x) b$ for all $x \in M \otimes N$. The argument for $L_{\psi}$ goes similarly.

Now, suppose $R_{\varphi}(x)=0$ for all $\varphi$ in $M_{*}$, then $\langle x, \varphi \otimes \psi\rangle=$ $\left\langle R_{\varphi}(x), \psi\right\rangle=0$ for $\varphi \in M_{*}$ and $\psi \in N_{*}$. Hence $\left\langle x, \sum_{i=1}^{n} \varphi_{i} \otimes \psi_{i}\right\rangle=$ 0 where $\varphi_{i} \in M_{*}$ and $\psi_{i} \in N_{*} \quad(i=1,2, \cdots, n)$. Since the family $\left\{\sum_{i=1}^{n} \varphi_{i} \otimes \psi_{i} \mid \varphi_{i} \in M_{*}, \psi_{i} \in N_{*}\right\}$ is dense in $(M \otimes N)_{*}$ (cf. [13]), we get $x=0$. Similarly the family $\left\{L_{\psi} \mid \psi \in N_{*}\right\}$ is also total in $M \otimes N$. This completes the proof.

We notice that the families $\left\{R_{\varphi} \mid \varphi \in M_{*}\right.$ and positive $\}$ and $\left\{M_{\psi} \mid \psi \in N_{*}\right.$ and positive $\}$ are also total. 
Recall that a factor $M$ (on a separable Hilbert space) has the property $L$ if there is a sequence $\left\{u_{n}\right\}$ of unitary elements in $M$ such that $\sigma$-weak limit $u_{n}=0$ and strong- limit $u_{n}^{*} a u_{n}=$ a for all $a \in M$.

Sakai proved that if one of the factors $M$ or $N$ is finite and has property $L$, then $M \otimes N$ has property $L$, and asked whether the restriction of finiteness could be dropped [4, Th. 6.4 and Remark 6.2]. Here we shall answer this question as an application of the above mappings.

Theorem 2. Let $M$ and $N$ be factors and suppose that $M$ or $N$ has property $L$; then $M \otimes N$ has property $L$.

Proof. Suppose $N$ has property $L$; then there is a sequence $\left\{u_{n}\right\}$ of unitary elements in $N$ such that $\sigma$-weak limit $u_{n}=0$ and strong-limit $u_{n}^{*} a u_{n}=a$ for all $a \in N$. Put $\widetilde{u}_{n}=1 \otimes u_{n}$, then $\left\{\widetilde{u}_{n}\right\}$ is a sequence of unitary elements in $M \otimes N$ and for $\phi \in M_{*}$ and $\psi \in N_{*}$,

$$
\lim _{n}<\tilde{u}_{n}, \varphi \otimes \psi>=\lim _{n}<1, \varphi><u_{n}, \psi>=0 .
$$

Hence, $\left.\lim _{n}<\widetilde{u}_{n}, \sum_{i=1}^{m} \varphi_{i} \otimes \psi_{i}\right\rangle=0$ where $\varphi_{i} \in M_{*}$ and $\psi_{i} \in N_{*}(i=$ $1,2, \cdots, m)$. Since $\left\{\widetilde{u}_{n}\right\}$ is uniformly bounded, this implies $\sigma$-weak $\operatorname{limit}_{n} \widetilde{u}_{n}=0$.

Next, take an arbitrary $x$ in $M \otimes N$, then for $\varphi \in M_{*}$ and $\psi \in N_{*}$ we get by Theorem 1

$$
\begin{aligned}
& \lim _{n}\left\langle\tilde{u}_{n}^{*} x \tilde{u}_{n}, \varphi \otimes \psi\right\rangle=\lim _{n}\left\langle R_{\varphi}\left(\tilde{u}_{n}^{*} x \tilde{u}_{n}\right), \psi\right\rangle \\
= & \lim _{n}\left\langle u_{n}^{*} R_{\varphi}(x) u_{n}, \psi\right\rangle=\left\langle R_{\varphi}(x), \psi\right\rangle=\langle x, \varphi \otimes \psi\rangle .
\end{aligned}
$$

Hence $\left.\quad \lim _{n}<\tilde{u}_{n}^{*} x \widetilde{u}_{n}, \quad \sum_{i=1}^{n} \varphi_{i} \otimes \psi_{i}\right\rangle=\left\langle x, \sum_{i=1}^{m} \varphi_{i} \otimes \psi_{i}\right\rangle \quad$ where $\varphi_{i} \in M_{*}$ and $\psi_{i} \in N_{*}(i=1,2, \cdots, m)$. Since $\left\{\tilde{u}_{n}^{*} x \tilde{u}_{n}\right\}$ is uniformly bounded, this implies $\sigma$-weak $\operatorname{limit}_{n} \widetilde{u}_{n}^{*} x \widetilde{u}_{n}=x$.

Let $\varphi$ be a normal positive functional on $M \otimes N$, then

$$
\begin{aligned}
& <\left(\widetilde{u}_{n}^{*} x \widetilde{u}_{n}-x\right)^{*}\left(\widetilde{u}_{n}^{*} x \widetilde{u}_{n}-x\right), \varphi> \\
= & <\widetilde{u}_{n}^{*} x^{*} x \widetilde{u}_{n}-\widetilde{u}_{n}^{*} x^{*} \widetilde{u}_{n} x-x^{*} \widetilde{u}_{n}^{*} x \widetilde{u}_{n}+x^{*} x, \varphi> \\
= & <\widetilde{u}_{n}^{*} x^{*} x \widetilde{u}_{n}, \varphi>-\left\langle\widetilde{u}_{n}^{*} x^{*} \widetilde{u}_{n} x, \varphi>-<x^{*} \widetilde{u}_{n}^{*} x \widetilde{u}_{n}, \varphi>\right. \\
& +<x^{*} x, \varphi>\Rightarrow<x^{*} x, \varphi>-<x^{*} x, \varphi>-<x^{*} x, \varphi> \\
& +<x^{*} x, \varphi>=0 .
\end{aligned}
$$

That is, strongest-limit $\widetilde{u}_{n}^{*} x \widetilde{u}_{n}=x$. and strong-limit $\widetilde{u}_{n}^{*} x \widetilde{u}_{n}=x$ for all $x \in M \otimes N$. Hence $M \otimes N$ has property $L$.

Let $M$ and $N$ act on $H$ and $K$. Denote by $\mathscr{B}(H)$ the algebra of all bounded linear operators on a Hilbert space $H$. Then $M \otimes N$ is naturally considered as the subalgebra of $\mathscr{B}(H) \otimes \mathscr{B}(K)=$ $\mathscr{B}(H \otimes K)$ and as is easily seen the mappings $R_{\varphi}$ and $L_{\psi}$ in $M \otimes N$ 
are nothing but the restrictions of those mappings $R_{\widetilde{\varphi}}\left(\widetilde{\mathscr{\varphi}} \in \mathscr{B}(H)_{*}\right)$ and $L_{\widetilde{\psi}}\left(\widetilde{\psi} \in \mathscr{B}(K)_{*}\right)$ in $\mathscr{B}(H) \otimes \mathscr{B}(K)$ where $\widetilde{\varphi}$ and $\widetilde{\psi}$ are extensions of $\varphi$ and $\psi$. Now the following question naturally arises. Let $\mathfrak{A}$ be a von Neumann algebra on $H \otimes K$ and suppose $\mathfrak{A}$ satisfies the following condition: $R_{\varphi}(\mathfrak{H}) \subset N$ for all $\varphi \in \mathscr{B}(H)_{*}$ and $L_{\psi}(\mathfrak{H}) \subset M$ for all $\psi \in \mathscr{B}(K)_{*}$, then what is the relation between $\mathfrak{A}$ and $M \otimes N$ ? All we know is that $\mathfrak{A}$ is contained in $M \otimes \mathscr{B}(K) \cap \mathscr{B}(H) \otimes N{ }^{1}$ In fact, let $x \in \mathfrak{A}$ and take an arbitrary element $a \in N^{\prime}$ then we get

$$
R_{\varphi}((1 \otimes a) x)=a R_{\varphi}(x)=R_{\varphi}(x) a=R_{\varphi}(x(1 \otimes a))
$$

for all $\varphi \in \mathscr{B}(H)_{*}$. Hence $(1 \otimes a) x=x(1 \otimes a)$ and $x \in\left(1 \otimes N^{\prime}\right)^{\prime}=$ $\mathscr{B}(H) \otimes N$. Similarly $x \in\left(M^{\prime} \otimes 1\right)^{\prime}=M \otimes \mathscr{B}(K)$. Thus

$$
\mathfrak{A} \subset M \otimes \mathscr{B}(K) \cap \mathscr{B}(H) \otimes N \text {. }
$$

Now let us consider the situation described in Theorem 1 . Putting $\widetilde{R}_{\varphi}(x)=1 \otimes R_{\varphi}(x)$ and $\widetilde{L}_{\psi}(x) L_{\psi}(x) \otimes 1$ we see that for commuting subalgebras $M \otimes 1$ and $1 \otimes N$ which generate $M \otimes N$ there are sufficiently many $\sigma$-weakly continuous $M \otimes 1$-module (resp. $1 \otimes N$-module) linear mappings from $M \otimes N$ to $M \otimes 1$ (resp. $1 \otimes N$ ) which induce $\sigma$-weakly continuous functional on each component algebra. We shall show that this situation completely determines the tensor product structure of von Neumann algebras. Namely

TheoRem 3. Let $\mathfrak{A}$ be a von Neumann algebra and $M$ and $N$ be subalgebras satisfying the following conditions:

(1) $\mathfrak{A}=R(M, N)$, i.e., $M$ and $N$ generate $\mathfrak{A}$,

(2) $M$ and $N$ commute with each other,

(3) There is a total family of $\sigma$-weakly continuous $N$-module mappings $\left\{R_{\alpha} \mid \alpha \in I\right\}$ from $\mathfrak{A}$ to $N$ such that $R_{\alpha}(a)=\lambda_{a}^{\alpha} 1$ for $a \in M$ where $\lambda_{a}^{\alpha}$ is a complex number associated with a.

Then $\mathfrak{A}$ is isomorphic to $M \otimes N$.

Proof. Take an element $\sum_{i=1}^{n} a_{i} b_{i}$ where $a_{i} \in M$ and $b_{i} \in N(i=$ $1,2, \cdots, n)$. We assert that the mapping

$$
\Phi: \sum_{i=1}^{n} a_{i} b_{i} \rightarrow \sum_{i=1}^{n} a_{i} \otimes b_{i}
$$

is well defined and one-to-one. So, let $\sum_{i=1}^{n} a_{i} b_{i}=0$. We may assume that $\left\{b_{i} \mid i=1,2, \cdots, n\right\}$ are linearly independent. Then, from the relation

1 According to the recent result [8] by Tomita about the general standard form of von Neumann algebras, the commutation theorem in the tensor products of von Neumann algebras follows as the corollary. Hence $\mathfrak{A} \subset M \otimes N$, i.e., $M \otimes N$ is the largest von Neumann algebra having $M$ and $N$ as its components in $H$ and $K$. A similar remark should also be added to Theorem 4 . 


$$
R_{\alpha}\left(\sum_{i=1}^{n} a_{i} b_{i}\right)=\sum_{i=1}^{n} R_{\alpha}\left(a_{i}\right) b_{i}=\sum_{i=1}^{n} \lambda_{a_{i}}^{\alpha} b_{i}=0,
$$

we get $\lambda_{a_{i}}^{\alpha}=0$ for $i=1,2, \cdots, n$ and $\alpha \in I$. Therefore $R_{\alpha}\left(a_{i}\right)=0$ for all $\alpha \in I$ and this means that $a_{i}=0$ for $i=1,2, \cdots, n$ and $\sum_{i=1}^{n} a_{i} \otimes b_{i}=$ 0 . Since the fact that $\sum_{i=1}^{n} a_{i} \otimes b_{i}=0$ implie $\sum_{i=1}^{n} a_{i} b_{i}=0$, the above result shows that $\Phi$ is a well defined one-to-one mapping. Therefore the $C^{*}$-algebra $C^{*}(M, N)$ generated by $M$ and $N$ is isomorphic to the $C^{*}$-tensor product of $M$ and $N$ with the compatible $C^{*}$-norm $\beta$ defined by

$$
\left\|\sum_{i=1}^{n} a_{i} \otimes b_{i}\right\|_{\beta}=\left\|\sum_{i=1}^{n} a_{i} b_{i}\right\|_{i} \quad \text { (cf. [7]). }
$$

Next consider the functional $\left\langle\alpha, \varphi_{\alpha}\right\rangle=\lambda_{a}^{\alpha}$ on $a \in M$ for a mapping $R_{\alpha}$. One easily sees that this is a $\sigma$-weakly continuous linear functional on $M$, i.e., $\varphi_{\alpha} \in M_{*}$. Now, for $\psi \in N_{*}$, we get

$$
\begin{aligned}
\left\langle\sum_{i=1}^{n} a_{i} b_{i},{ }^{t} R_{\alpha}(\psi)\right\rangle & =\sum_{i=1}^{n}\left\langle R_{\alpha}\left(a_{i} b_{i}\right), \psi\right\rangle \\
=\sum_{i=1}^{n}\left\langle R_{\alpha}\left(a_{i}\right) b_{i}, \psi\right\rangle & =\sum_{i=1}^{n}\left\langle a_{i}, \varphi_{\alpha}\right\rangle\left\langle b_{i}, \psi\right\rangle \\
= & \left\langle\sum_{i=1}^{n} a_{i} \otimes b_{i}, \varphi_{\alpha} \otimes \psi\right\rangle .
\end{aligned}
$$

Hence for $x \in C^{*}(M, N)$

$$
\left\langle R_{\alpha}(x), \psi\right\rangle=\left\langle x,{ }^{t} R_{\alpha}(\psi)\right\rangle=\left\langle\Phi(x), \varphi_{\alpha} \otimes \psi\right\rangle \cdots\left({ }^{*}\right) .
$$

Therefore if $\left\langle\Phi(x), \varphi_{\alpha} \otimes \psi\right\rangle=0$ for all $\varphi_{\alpha}$ and $\psi \in N_{*}$, then $R_{\alpha}(x)=0$ for all $\alpha \in I$ and $x=0$. That is, $\Phi(x)=0$. Thus in $M \otimes_{\beta} N$ the family of all product functionals $\varphi \otimes \psi\left(\varphi \in M^{*}, \psi \in N^{*}\right)$ is total, hence the norm $\beta$ must coincide with Turumaru's $\alpha$-norm and $C^{*}(M, N) \cong M \otimes_{\alpha} N$. (see [7, Th. 2]).

Let $V=$ Linear span of $\left\{{ }^{t} R_{\alpha}(\psi): \alpha \in I, \psi \in N_{*}\right\}$. Since $\left\{R_{\alpha} \mid \alpha \in I\right\}$ is total in $\mathfrak{A}, V$ is uniformly dense in $\mathfrak{A}_{*}$. On the other hand, let $V^{\prime}=$ linear span of $\left\{\varphi_{\alpha} \otimes \psi \mid \alpha \in I, \psi \in N_{*}\right\}$, then $V^{\prime}$ is also uniformly dense in $(M \otimes N)_{*}$ and by the equality $\left(^{*}\right)$ we get ${ }^{t} \Phi\left[V^{\prime} \mid M \otimes_{\alpha} N\right]=V \mid C^{*}(M, N)$ where $V^{\prime} \mid M \otimes_{\alpha} N$ and $V \mid C^{*}(M, N)$ are the restrictions of elements in $V^{\prime}$ and $V$ to $M \otimes_{\alpha} N$ and $C^{*}(M, N)$ respectively. Now by Kaplansky's density theorem, $V$ and $V^{\prime}$ are isometric to $V \mid C^{*}(M, N)$ and $V^{\prime} \mid M \otimes_{\alpha} N$, so that ${ }^{t} \Phi$ induces the isometry between $V^{\prime}$ and $V$, hence the isometry $\rho$ between $(M \otimes N)_{*}$ and $\mathfrak{U}_{*}$. It is not difficult to see that ${ }^{t} \rho$ is the extended isomorphism of $\Phi$ between $\mathfrak{U}$ and $M \otimes N$. This completes the proof.

In the above theorem, the case where $\mathfrak{A}$ is a finite factor is due 
to Nakamura [2] and the case where $\mathfrak{Y}$ is a (general) factor is proved in Takesaki [6].

Our next result is somewhat different from those treated above and is included essentially in Lemma 2.3 and in the proof of Theorem 3.2 of the author's joint work [1] with Hakeda. However, it may be useful to reformulate these results in the simple form shown below. We give its proof for completeness.

THEOREM 4. Let $M_{1}$ and $N_{1}$ be von Neumann algebras on $H$ and $K$ and $M_{2}$ and $N_{2}$ be their von Neumann subalgebras respectively. Suppose there are projections of norm one $\pi_{1}$ and $\pi_{2}$ from $M_{1}$ to $M_{2}$ and from $N_{1}$ to $N_{2}$. Then there is a projection of norm one

$$
\pi: M_{1} \otimes \mathscr{B}(K) \cap \mathscr{B}(H) \otimes N_{1} \rightarrow M_{2} \otimes \mathscr{B}(K) \cap \mathscr{B}(H) \otimes N_{2}
$$

such that $\pi(a \otimes b)=\pi_{1}(a) \otimes \pi_{2}(b)$ where $a \in M_{1}$ and $b \in N_{1}$.

It is known that in the above case there is a unique projection of norm one $\pi_{1} \otimes \pi_{2}$ from $M_{1} \otimes_{\alpha} N_{1}$ to $M_{2} \otimes_{\alpha} N_{2}$ such that $\pi_{1} \otimes \pi_{2}(a \otimes b)=$ $\pi_{1}(a) \otimes \pi_{2}(b)$, and if $\pi_{1}$ and $\pi_{2}$ are $\sigma$-weakly continuous it can be also shown that we can extend the above $\pi_{1} \otimes \pi_{2}$ to the $\sigma$-weak continuous projection of norm one from $M_{1} \otimes N_{1}$ to $M_{2} \otimes N_{2}$ which is a posteriori unique (cf. [10]). However, it is the crucial point of the above theorem that even if we lack the condition of $\sigma$-weak continuity of $\pi_{1}$ and $\pi_{2}$ we get still the extension of $\pi_{1} \otimes \pi_{2}$ to the algebra $M_{1} \otimes \mathscr{\mathscr { B }}(K) \cap \mathscr{B}(H) \otimes N_{1}$.

Proof of the Theorem. Let $\left\{e_{i} \mid i \in I\right\}$ be the family of orthogonal minimal projections in $\mathscr{B}(K)$ corresponding to the orthogonal basis in $K$. Put $\widetilde{e}_{i}=1 \otimes e_{i}, \quad e_{J}=\sum_{i \in J} e_{i}$ and $\widetilde{e}_{J}=1 \otimes e_{J}=\sum_{i \in J} \widetilde{e}_{i}$ where $J$ is a finite subset of $I$. Then

$$
\widetilde{e}_{J} M_{1} \otimes \mathscr{B}(K) \widetilde{e}_{J}=M_{1} \otimes e_{J} \mathscr{B}(K) e_{J}=M_{1} \otimes_{\alpha} e_{J} \mathscr{B}(K) e_{J}
$$

(the last equality holds, since $e_{J} \mathscr{B}(K) e_{J}$ is a finite dimensional algebra). Let $\pi_{J}$ be the projection of norm one from $M_{1} \otimes_{\alpha} e_{J} \mathscr{B}(K) e_{J}$ to $M_{2} \otimes_{\alpha} e_{J} \mathscr{B}(K) e_{J}$ defined by $\pi_{J}(a \otimes b)=\pi_{1}(a) \otimes b$ where $a \in M_{1}$ and $b \in e_{J} \cdot \mathscr{B}(K) e_{J}$ (cf. [10, Th. 1]) and put $\pi_{J}^{1}(x)=\pi_{J}\left(\widetilde{e}_{J} x \widetilde{e}_{J}\right)$ for $x \in M_{1} \otimes \mathscr{B}(K)$. Then $\left\{\pi_{\nu}^{1}(x) \mid J\right.$ is a finite subset of $\left.I\right\}$ is a family of elements in $M_{2} \otimes \mathscr{B}(K)$ bounded by $\|x\|$. Put $\pi^{1}(x)=\operatorname{Lim}_{J} \pi_{J}^{1}(x)$ (operator Banach limit in the sense of Schwartz [5] with respect to the subsets $J)$. By the property of the operator Banach limit shown in [5], we have

$$
\pi^{1}(x) \in M_{2} \otimes \mathscr{B}(K) \text { and }\left\|\pi^{1}(x)\right\| \leqq\|x\|
$$


Moreover, for $x \in M_{2} \otimes \mathscr{B}(K), x=\sigma$-weak limit $\widetilde{e}_{J} x \widetilde{e}_{J}$ implies

$$
\begin{gathered}
\operatorname{Lim}_{J} \pi_{J}^{1}(x)=\operatorname{Lim}_{J} \pi_{J}\left(\widetilde{e}_{J} x \widetilde{e}_{J}\right)=\operatorname{Lim}_{J} \widetilde{e}_{J} x \widetilde{e}_{J} \\
=\sigma \text {-weak limit } \widetilde{e}_{J} x \widetilde{e}_{J}=x .
\end{gathered}
$$

Therefore $\pi^{1}$ is a projection of norm one from $M_{1} \otimes \mathscr{B}(K)$ to $M_{2} \otimes \mathscr{B}(K)$. Take an element $a \otimes b \in M_{1} \otimes \mathscr{B}(K)$. We have

$$
\begin{aligned}
\pi^{1}(a \otimes b) & =\operatorname{Lim}_{J} \pi_{J}\left(a \otimes e_{J} b e_{J}\right)=\operatorname{Lim}_{J} \pi_{1}(a) \otimes e_{J} b e_{J} \\
& =\sigma \text {-weak } \operatorname{limit}_{J} \pi_{1}(a) \otimes e_{J} b e_{J}=\pi_{1}(a) \otimes b
\end{aligned}
$$

Similarly we get a projection of norm one $\pi^{2}$ from $\mathscr{B}(H) \otimes N_{1}$ to $\mathscr{Q}(H) \otimes N_{2}$ such that $\pi^{2}(a \otimes b)=a \otimes \pi_{2}(b)$.

Now take an element $x \in M_{1} \otimes \mathscr{B}(K) \cap \mathscr{B}(H) \otimes N_{1}$. For arbitrary $y \in M_{1}^{\prime} \otimes 1$ we get, by Theorem 1 in Tomiyama [9],

$$
y \pi^{2}(x)=\pi^{2}(y x)=\pi^{2}(x y)=\pi^{2}(x) y
$$

because $M_{1}^{\prime} \otimes 1 \subset \mathscr{B}(H) \otimes N_{2}$ and $\left(M_{1}^{\prime} \otimes 1\right)^{\prime}=M_{1} \otimes \mathscr{B}(K)$. Hence $\pi^{2}(x) \in M_{11} \otimes \mathscr{B}(K) \cap \mathscr{B}(H) \otimes N_{2}$. Therefore, put $\pi(x)=\pi^{1} \pi^{2}(x)_{\text {a }}$ and take an element $y \in 1 \otimes N_{2}^{\prime}$. Since $1 \otimes N_{2}^{\prime} \subset M_{2} \otimes \mathscr{B}(K)$ we get again by [9, Th. 1]

$$
y \pi(x)=y \pi^{1} \pi^{2}(x)=\pi^{1}\left(y \pi^{2}(x)\right)=\pi^{1}\left(\pi^{2}(x) y\right)=\pi(x) y .
$$

Thus $\pi(x) \in M_{2} \otimes \mathscr{B}(K) \cap \mathscr{B}(H) \otimes N_{2}$ and it is clear that this mapping $\pi$ is a projection of norm one from $M_{1} \otimes \mathscr{B}(K) \cap \mathscr{B}(H) \otimes N_{1}$ to $M_{2} \otimes \mathscr{B}(K) \cap \mathscr{B}(H) \otimes N_{2}$. Finally for $a \otimes b \in M_{1} \otimes N_{1}$ we have

$$
\pi(a \otimes b)=\pi^{1} \pi^{2}(a \otimes b)=\pi^{1}\left(a \otimes \pi_{2}(b)\right)=\pi_{1}(a) \otimes \pi_{2}(b) .
$$

This completes the proof.

\section{REFERENCES}

1. J. Hakeda and J. Tomiyama, On some extension properties of von Neumann algebras, Tôhoku Math. J. 19 (1967), 315-323.

2. M. Nakamura, On the direct product of finite factors, Tôhoku Math. J. 6 (1954), 205-207.

3. L. Pukánszky, Some examples of factors, Publ. Math. 4 (1956), 135-156.

4. S. Sakai, The theory of $W^{*}$-algebras, Yale Univ., 1962.

5. J. Schwartz, Two finite non-hyperfinite, non-isomorphic factors, J. Pure and Applied Math. 16 (1963), 19-26.

6. M. Takesaki, On the direct product of $W^{*}$-factors, Tôhoku Math. J. 10 (1958), 116-119.

7. - On the cross-norm of the direct product of $C^{*}$-algebras, Tohoku Math. J. 16 (1964), 111-122.

8. M. Tomita, Standard forms of von Neumann algebros (to appear)

9. J. Tomiyama, On the projection of norm one in $W^{*}$-algebras, Proc. Japan Acad. 33 (1957), 608-612. 
10. - On the product projection of norm one in the direct product of operator algebras, Tôhoku Math. J. 11 (1959), 305-313.

11. - Applications of Fubini type theorem to the tensor product of $C^{*}$-algebras, Tôhoku Math. J. 19 (1967), 213-226.

12. T. Turumaru, On the direct product of operator algebras, I. Tôhoku Math. J. 4 (1952), 242-251.

13. - On the direct product of operator algebras, III, Tôhoku Math. J. 6 (1954), 208-211.

Received January 8, 1968. The preparation of this paper was supported in part by Grant No. GP-6726.

UNiversity of CALIFornia, Los ANGEles and

YAMAGATA UNIVERSITY

YAMAGATA, JAPAN 


\section{PACIFIC JOURNAL OF MATHEMATICS}

\section{EDITORS}

H. ROYDEN

Stanford University

Stanford, California

Richard Pierce

University of Washington

Seattle, Washington 98105
J. DugundJI

Department of Mathematics

University of Southern California

Los Angeles, California 90007

BASIL GORDON

University of California

Los Angeles, California 90024

\section{ASSOCIATE EDITORS}

E. F. BECKENBACH

B. H. NeUmanN

F. WOLF

K. YOSHIDA

\section{SUPPORTING INSTITUTIONS}

UNIVERSITY OF BRITISH COLUMBIA

CALIFORNIA INSTITUTE OF TECHNOLOGY

UNIVERSITY OF CALIFORNIA

MONTANA STATE UNIVERSITY

UNIVERSITY OF NEVADA

NEW MEXICO STATE UNIVERSITY

OREGON STATE UNIVERSITY

UNIVERSITY OF OREGON

OSAKA UNIVERSITY

UNIVERSITY OF SOUTHERN CALIFORNIA
STANFORD UNIVERSITY

UNIVERSITY OF TOKYO

UNIVERSITY OF UTAH

WASHINGTON STATE UNIVERSITY

UNIVERSITY OF WASHINGTON

$* * \quad * \quad *$
AMERICAN MATHEMATICAL SOCIETY
CHEVRON RESEARCH CORPORATION
TRW SYSTEMS
NAVAL WEAPONS CENTER




\section{Pacific Journal of Mathematics}

Vol. 30, No. $1 \quad$ September, 1969

William Wells Adams, Simultaneous diophantine approximations and cubic irrationals ..................................... 1

Heinz Bauer and Herbert Stanley Bear, Jr., The part metric in convex

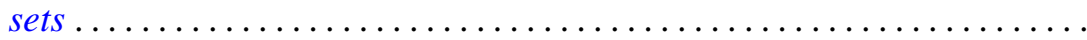

L. Carlitz, A note on exponential sums ...................... 35

Vasily Cateforis, On regular self-injective rings ................. 39

Franz Harpain and Maurice Sion, A representation theorem for measures on infinite dimensional spaces ......................... 47

Richard Earl Hodel, Sum theorems for topological spaces .............. 59

Carl Groos Jockusch, Jr. and Thomas Graham McLaughlin, Countable

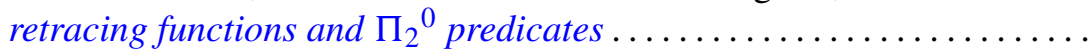

Bjarni Jónsson and George Stephen Monk, Representations of primary

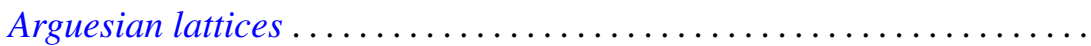

Virginia E. Walsh Knight, A continuous partial order for Peano continua...................................... 141

Kjeld Laursen, Ideal structure in generalized group algebras ........... 155

G. S. Monk, Desargues' law and the representation of primary lattices . . . 175

Hussain Sayid Nur, Singular perturbation of linear partial differential equation with constant coefficients ..........................

Richard Paul Osborne and J. L. Stern, Covering manifolds with cells ... 201

Keith Lowell Phillips and Mitchell Herbert Taibleson, Singular integrals in several variables over a local field...

James Reaves Smith, Local domains with topologically $T$-nilpotent radical....

Donald Platte Squier, Elliptic differential equations with discontinuous coefficients .................................

Tae-il Suh, Algebras formed by the Zorn vector matrix...

Earl J. Taft, Ideals in admissible algebras . .................... 259

Jun Tomiyama, On the tensor products of von Neumann algebras........ 263

David Bertram Wales, Uniqueness of the graph of a rank three group ..... 271

Charles Robert Warner and Robert James Whitley, A characterization of regular maximal ideals ......................... 